\section{Reprodutibilidade do teste de uma repetição máxima em exercícios de força com pesos livres}

\section{Multiples sessions of one-repetition maximum test in exercises with free weights}

\author{
Paulo Fernando Marinho de Lima ${ }^{1}$ \\ Rodrigo Cappato de Araújo ${ }^{1}$ \\ Breno Quintella Farah ${ }^{1}$ \\ Bruno Remígio Cavalcante ${ }^{2}$ \\ Marcos André Moura dos Santos ${ }^{2}$ \\ Raphael Mendes Ritti-Dias ${ }^{1}$
}

\section{Resumo}

O objetivo do presente estudo foi verificar a quantidade de sessões necessárias para determinação da carga máxima em testes múltiplos de uma repetição máxima nos exercícios de força com pesos livres. Dezessete homens (22,5 $\pm 3,5$ anos), não praticantes de musculação há quatro meses, fizeram quatro sessões de teste de 1-RM em cinco exercícios: supino reto, remada curvada, elevação frontal, rosca direta de bíceps e tríceps testa, respectivamente. Para o tratamento dos dados foi utilizada a ANOVA one way, seguida do teste post hoc de Nerwman-Keuls, com p<0.05. A estabilização da carga para os exercícios supino reto, rosca direta de bíceps e tríceps testa ocorreu entre a 3 e 4 sessões de teste. Para os exercícios remada curvada e elevação frontal quatro sessões não foram suficientes para encontrar uma medida acurada de força máxima. Sugere-se a realização de três sessões de teste para os exercícios supino horizontal, rosca direta de bíceps e tríceps testa e de pelo menos quatro sessões para os exercícios remada curvada e elevação frontal.

\section{Palavras-chave}

Treinamento de resistência; Força muscular; Homens.

\begin{abstract}
The aim of this study was to determine the amount of sessions needed to determine the maximum load in multiple tests of one-repetition maximum $(1-R M)$ in the strength training with free weights. 17 men (22.5 \pm 3.5 years), not trained in strength four months ago, participated of four test sessions of 1-RM in five exercises: bench press, seated rowing, front raise, arm curl and lying down triceps extension, respectively. For the treatment of the data was used one-way ANOVA followed by post hoc Newman-Keuls tests, $P<0.05$. The stabilization of the load for the bench press, arm curl and lying down triceps extension occurred between $3^{\text {rd }}$ and $4^{\text {st }}$ test sessions. For the seated rowing and front raise, four sessions were not enough to find an accurate measure of maximum strength. It is suggested make three test sessions for exercises bench press, arm curl and lying down triceps extension and at least four sessions for exercises seated rowing and front raise.
\end{abstract}

\section{Keywords}

Resistance training; Muscle strength; Men.
Rev Bras Ativ Fis Saúde p. 378-386 DOI:

http://dx.doi.org/10.12820/rbafs.v.18n3p378

1 Programa Associado de Pós-graduação em Educação Física UPE/UFPB, Recife, PE, Brasil

2 Escola Superior de Educação Física da Universidade de Pernambuco (ESEF-UPE), Recife, $P E$, Brasil 


\section{INTRODUÇÃO}

Nos últimos anos, o treinamento de força tem sido recomendado para melhoria da aptidão musculoesquelética quando sua utilização envolve a aplicação de cargas elevadas a fim de proporcionar maiores adaptações como aumento da força e massa muscular ${ }^{1}$. Nesta perspectiva, o monitoramento da evolução das cargas utilizadas em um programa de treinamento de força é extremamente interessante para identificar alterações induzidas por esta modalidade.

O teste de uma repetição máxima (1-RM) tem sido o método mais utilizado para avaliação da carga máxima em movimentos isoinerciais em pesquisas científicas. Todavia, sua utilização requer alguns cuidados metodológicos, dentre os quais a familiarização prévia ao teste têm sido um dos mais estudados. De fato, está bem evidenciado na literatura que a falta de familiarização com os procedimentos do teste de 1-RM pode comprometer os resultados obtidos ${ }^{2}$.

No entanto, o número de sessões necessárias para a familiarização ao teste de 1-RM tem divergido consideravelmente entre os estudos. Por exemplo, Cronin et al., ${ }^{3}$ verificaram a necessidade de três sessões de familiarização no exercício agachamento na barra guiada, ao passo que Ritti-Dias et al. ${ }^{4}$ observaram a necessidade de apenas duas sessões para esse mesmo exercício. Como o nível de treinamento entre os sujeitos dos estudos parece ser similar, é possível que a discrepância entre os resultados dos estudos estejam associados aos diferentes equipamentos utilizados para a realização do teste.

Diante disso, a utilização de exercícios com pesos livres pode fornecer informações com maior aplicabilidade prática, uma vez que permite a extrapolação dos resultados obtidos sem o viés das características específicas do equipamento. Todavia, até o presente momento, poucos estudos têm analisado a familiarização em exercícios com pesos livres e aqueles que se propuseram a essa investigação analisaram apenas poucos exercícios. Dessa forma, o objetivo deste estudo foi verificar a quantidade de sessões de familiarização necessárias para determinação da força máxima em testes múltiplos de 1-RM nos exercícios de força com pesos livres.

\section{MATERIAIS E MÉTODOS}

\section{Amostra}

Participaram voluntariamente do estudo 17 jovens universitários do sexo masculino, com experiência prévia em treinamento de força (idade: $22,5 \pm 3,5$ anos; massa corporal: $66,4 \pm 5,7 \mathrm{~kg}$; estatura: $173,6 \pm 5,3 \mathrm{~cm}$; índice de massa corporal: $22,0 \pm 1,6$ $\mathrm{kg} / \mathrm{m}^{2}$ ). Os sujeitos não deveriam estar envolvidos recentemente com o treinamento sistemático de força (pelo menos: quatro meses), estabelecido como critério de inclusão no estudo. Todos os indivíduos assinaram o Termo de Consentimento Livre e Esclarecido. Este estudo foi aprovado pelo Comitê de Ética em Pesquisa com Seres Humanos da Universidade de Pernambuco de acordo com as normas estabelecidas pela Comissão Nacional de Ética e Pesquisa (CONEP), Resolução No. 196/96, sobre pesquisa envolvendo seres humanos (0203.0.097.000-08).

\section{PROCEDIMENTOS METODOLÓGICOS}

\section{Sessão de padronização}

Previamente ao início da realização dos testes, foram executadas duas sessões de padronização que tinham como objetivo realizar os ajustes necessários para exe- 
cução apropriada dos exercícios. Foram observadas e registradas as localizações do posicionamento das mãos nas barras, angulação do quadril e o posicionamento dos pés. Os sujeitos foram orientados a executarem o movimento com a máxima amplitude possível em cada exercício proposto. A sessão foi composta de três séries com 15 repetições, utilizando apenas a barra livre como sobrecarga. O intervalo de recuperação entre as séries e entre os exercícios foi de 90 segundos. Após o ajustamento dos exercícios, os testes de 1-RM seriam realizados após um período de 24 horas.

Todos os indivíduos foram orientados a não praticarem atividades físicas e manterem padrões semelhantes de sono e alimentação ao longo das 24 horas antecedentes à realização dos testes para determinação da carga máxima. Além disso, eles foram aconselhados a não utilizar substâncias ergogênicas nos dias dos testes.

\section{Testes de 1-RM}

Todos os sujeitos foram submetidos a quatro sessões de testes de 1-RM, nos exercícios supino horizontal, remada curvada, elevação frontal, rosca direta e tríceps na testa, respectivamente sendo estabelecido um intervalo de 48 horas entre cada sessão. Cada um dos exercícios foi precedido de um aquecimento (12 a 15 repetições), com aproximadamente $50 \%$ da carga estimada arbitrariamente para primeira tentativa do teste de 1- RM. Após dois minutos de repouso os testes eram iniciados.

Os indivíduos foram encorajados a realizar duas repetições com a carga imposta nas três tentativas para cada exercício. Caso as duas repetições fossem completadas na primeira tentativa, ou mesmo se não fosse completada sequer uma única repetição, uma segunda tentativa era realizada, após um intervalo de recuperação de três a cinco minutos, com uma carga superior (primeira possibilidade) ou inferior (segunda possibilidade) àquela empregada anteriormente. Tal procedimento foi repetido em uma terceira e última tentativa caso não houvesse sido determinada uma única repetição máxima ${ }^{5}$. A carga de 1-RM registrada foi aquela em que o sujeito foi capaz de realizar uma única repetição. Para todos os exercícios, a repetição só foi considerada como correta, quando houve a execução completa da mesma, tanto em sua fase concêntrica, quanto sua fase excêntrica ${ }^{5}$. Os registros foram feitos individualmente, e em seguida, enviados para uma segunda pessoa que fez a tabulação dos dados. O posicionamento dos exercícios seguiu a seguinte padronização:

- O exercício supino horizontal foi realizado em um banco horizontal com barra livre. O posicionamento das mãos na barra era padronizado de maneira que os polegares estavam alinhados com o ombro no momento em que a barra estava no suporte. Era considerado um movimento completo quando o indivíduo descia a barra até que esta tocasse o peito e subia a barra até a extensão da articulação do cotovelo.

- No exercício remada curvada, os sujeitos posicionavam as mãos em pronação na barra, de tal forma que os polegares se alinhavam com os ombros quando estava em repouso com a barra ao nível dos joelhos. A coluna permaneceu em posição perpendicular à amplitude de movimento em uma posição neutra a $45^{\circ}$ para o chão, sendo esta verificada através de um goniômetro. Para a execução ser considerada válida os sujeitos deveriam trazer a barra do nível do joelho até a região abdominal e retornando a posição inicial, mantendo os cotovelos junto ao corpo.

- O exercício elevação frontal foi executado com barra livre. As mãos estavam em pronação na barra, de tal forma que os polegares se alinhavam com os ombros quando estava em repouso com a barra ao nível das coxas. Para a execução ser 
considerada válida os sujeitos deveriam levantar a barra do nível da coxa até o nível dos ombros e retornar da posição inicial, mantendo os braços estendidos.

- O exercício rosca direta de bíceps foi executado com as costas apoiadas em uma parede e com os joelhos semiflexionados. As mãos estavam em supinação na barra, de tal forma que os polegares estavam alinhados com o ombro quando permanecia em repouso, com a barra ao nível das coxas. Para a execução ser considerada válida os sujeitos deveriam realizar a flexão do cotovelo, partindo da extensão total dos cotovelos e indo até a flexão total, sem o movimento das pernas ou das costas.

- O exercício tríceps testa os sujeitos posicionavam as mãos em pronação na barra, de tal forma que os dedos mínimos se alinhavam com o ombro quando estava em repouso com a barra no suporte de descanso. Para a execução ser considerada válida os sujeitos deveriam abaixar a barra até atingir um nível próximo da testa e retornar a posição inicial, mantendo os braços imóveis em $90^{\circ}$ com o corpo. A distância constante entre os cotovelos foi mantida durante todo o movimento.

\section{Análise estatística}

Os testes de Shapiro-Wilk e de Levene foram realizados para verificar a normalidade e homogeneidade dos dados, respectivamente. Os resultados são apresentados em média e desvio padrão. Para verificar a estabilização da carga de 1-RM ao longo das quatro sessões de teste, utilizou-se a ANOVA de um fator para medidas repetidas, seguido do teste de post hoc de Nerwman-Keuls. A plotagem de Bland-Altman foi utilizada para verificar a concordância entre os valores encontrados nas sessões em que ocorreu a suposta estabilização, em cada um dos exercícios investigados e os respectivos limites de concordância de $95 \%$ ( \pm 1,96 desvio padrão). Para avaliação da reprodutibilidade interdias foi utilizado o Coeficiente de Correlação de Intraclasse (CCI). Os valores de CCI foram interpretados como: pobre se $<0.40$, moderada se variou entre 0,40 e 0,75 , e excelente se $>0,75$, de acordo com a escala de níveis de confiabilidade proposta por Fleiss ${ }^{6}$. Os dados foram analisados utilizando os pacotes estatísticos Statistica (versão 7.0) (Statsoft, EUA,1995) e GraphPad Prism versão 3.0 (GraphPad Software Inc., EUA, 2003). O nível de significância adotado em todas as análises foi de $5 \%$.

\section{RESULTADOS}

Todos os sujeitos realizaram com sucesso as quatro sessões de teste de 1-RM nos cinco exercícios propostos. Além disso, nenhuma intercorrência foi observada durante a realização dos testes. Na tabela 1 são apresentados os coeficientes de correlação intraclasse entre as quatro sessões de teste nos cinco exercícios analisados. Os valores de CCI variaram de 0,81 a 0,98, o que demonstra excelentes níveis de reprodutibilidade do teste de $1-\mathrm{RM}$ para todos os exercícios analisados.

Tabela 1 - Coeficiente de correlação intraclasse da carga de 1-RM nos cinco exercícios.

\begin{tabular}{lc}
\hline Exercícios & Coeficiente de Correlação Intraclasse (IC95\%) \\
\hline Supino horizontal & $0,98(0,96-0,99)$ \\
\hline Remada curvada & $0,88(0,77-0,95)$ \\
\hline Elevação frontal & $0,86(0,74-0,94)$ \\
\hline Rosca direta de bíceps & $0,96(0,91-0,98)$ \\
\hline Tríceps testa & $0,81(0,67-0,92)$ \\
\hline
\end{tabular}

IC95\%- Intervalo de confiança 95\%. 
Nas figuras 1 e 2 são apresentados os valores da carga máxima obtidos em cada exercício em cada sessão de teste. Nos exercícios supino reto (figura - 1A), rosca direta bíceps (figura - 1B) e tríceps testa (figura - 1C), foram observadas diferenças significantes entre as sessões 1 e 2 e as demais sessões, sendo que não foram encontradas diferenças significantes entre as sessões 3 e 4 (supino reto $\mathrm{p}=0,19$; rosca direta bíceps $-\mathrm{p}=0,74$; tríceps $-\mathrm{p}=0,06$ ). Já nos exercícios remada curvada (Figura 2A) e elevação frontal (figura 2B) foram encontradas diferenças significantes entre as sessões de teste (remada curvada - 1 vs. 2 : $p=0,021 ; 1$ vs. 3 : $p=0,003 ; 1$ vs. $4: p<0,001 ; 2$ vs. $3: p=0,287 ; 2$ vs. $4: p=0,017 ; 3$ vs. $4: p=0,079$; elevação frontal -1 vs. 2 : $p=0,075 ; 1$ vs. 3 : $p=0,001 ; 1$ vs. $4: p<0,001 ; 2$ vs. $3: p=0,049$; 2 vs. $4: \mathrm{p}<0,001 ; 3$ vs. $4: \mathrm{p}=0,019)$, indicando que ao final da quarta sessão ainda não existia estabilização da carga.
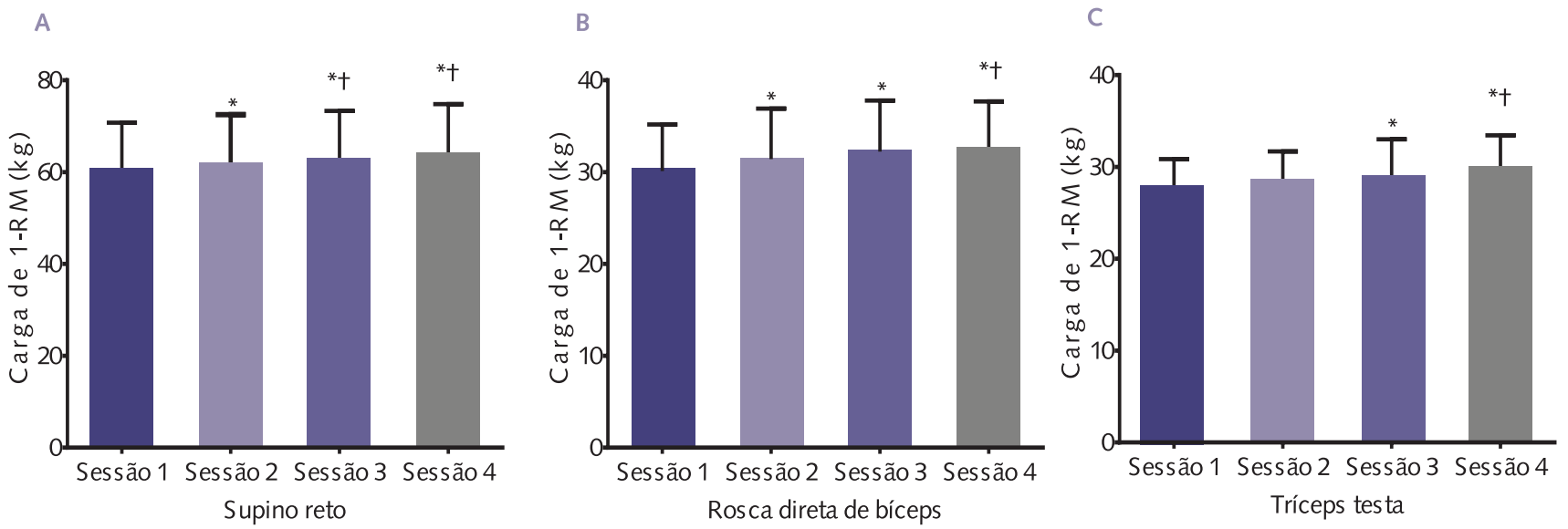

Figura 1 - Carga máxima ao longo das quatro sessões de teste nos exercícios supino reto, rosca direta de bíceps e tríceps testa. * Diferença significante em relação a sessão 1; e † Diferença significante em relação a sessão 2; e ‡ Diferença significante em relação a sessão 3.
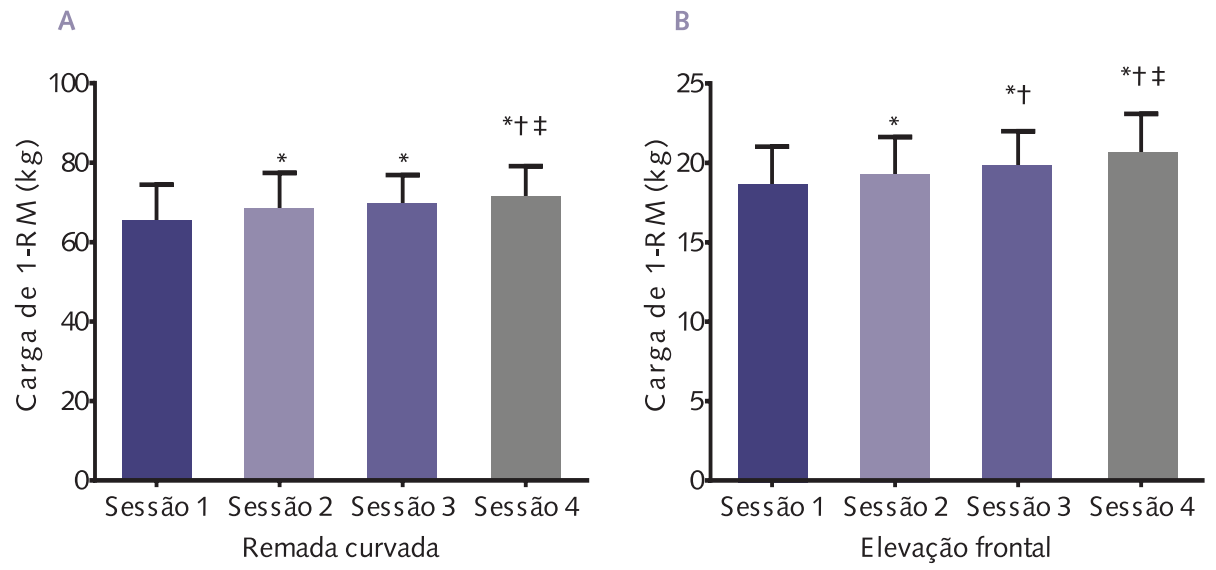

Figura 2 - Carga máxima ao longo das quatro sessões de teste nos exercícios remanada curvada e elevação frontal. * Diferença significante em relação a sessão 1; e † Diferença significante em relação a sessão 2; e ₹ Diferença significante em relação a sessão 3.

$\mathrm{Na}$ figura 3 são apresentadas as análises de concordância entre as sessões dos exercícios, em que foi verificada estabilização da carga, por meio da Plotagem de Bland-Altman. Nos exercícios supino reto (figura 3A), rosca direta bíceps (figura 3B) e tríceps testa (figura 3C), foi verificada um boa concordância entre as sessões 3 e 4, respectivamente, uma vez que a maioria dos casos os valores encontram-se próximos de zero e dentro dos limites estabelecidos. 

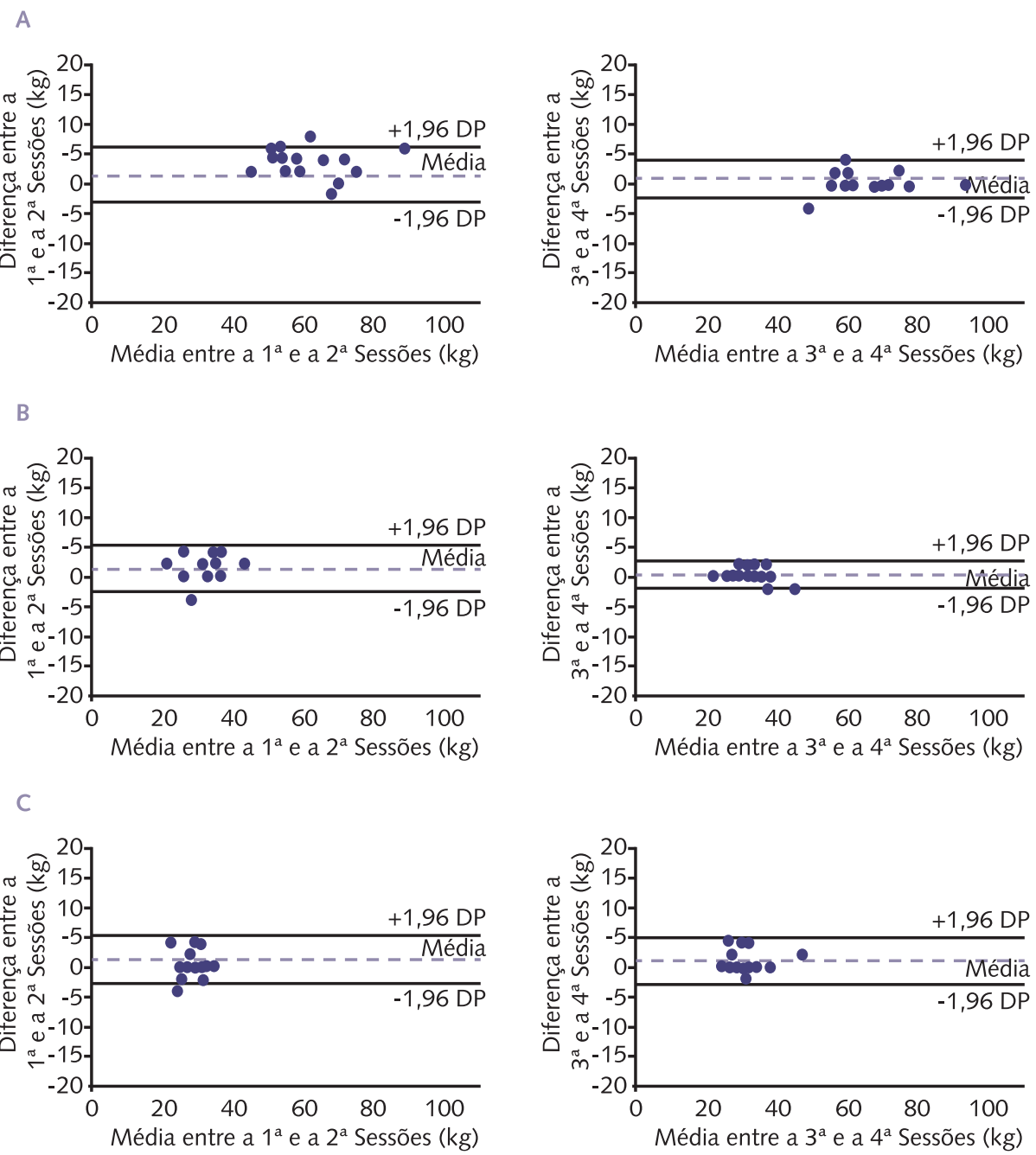

Figura 3 - Concordância entre as sessões obtidas no teste de 1-RM nos exercício supino reto (figura A), rosca direta bíceps (figura B) e tríceps testa (figura C). Abscissa: diferenças entre as sessões de 1-RM. Ordenada: média entre as sessões.

\section{DISCUSSÃO}

O objetivo do presente estudo foi verificar a quantidade de sessões necessárias para verificar a medida acurada da força máxima em testes de 1-RM para exercícios com peso livre. Esperava-se que com no máximo de quatro sessões teste de 1-RM ocorreria à estabilização da carga em todos os exercícios, no entanto, este resultado se mostrou evidente nos exercícios supino reto, rosca direta bíceps e tríceps testa.

Já está bem descrito na literatura a importância da familiarização ao teste de 1-RM para obtenção acurada da força máxima ${ }^{7-12}$, de forma que a falta destes procedimentos pode comprometer a qualidade dos resultados obtidos. Assim como descrito previamente na literatura ${ }^{9,13}$, foram encontrados excelentes níveis de reprodutibilidade $(\mathrm{CCI} \geq 0,81)$ do teste de $1-\mathrm{RM}$ em todos os exercícios. Entretanto, em alguns casos é necessária a combinação de diferentes métodos estatísticos, pois o CCI usado de forma isolado pode apresentar enganosos níveis de reprodutibilidade. Isso acontece, pois quando se mede uma variável em um grupo de diferentes sujeitos, espera-se que as medidas apesar de diferentes fiquem relativamente próximas da média, produzindo assim, altos valores de CCI. O uso de métodos que analisam a variância e concordância dos dados surge como opção 
mais confiável e tem sido frequentemente utilizado em outros estudos 9,12-15. Nesse sentido, a comparação e discussão dos dados do presente estudo serão pautadas com base nos resultados destes métodos.

Vários estudos verificaram diferentes quantidades de sessões para diferentes exercícios de força, podendo a quantidade de sessões variar de 1 a 9 sessões ${ }^{2,3}$, 7,9,10,13-17 dependendo da característica dos sujeitos. Dias et al. ${ }^{9}$, em estudo que avaliou dezoito mulheres adultas, identificou a necessidade de 2 a 3 sessões para os exercícios supino reto e rosca direta de bíceps para estabilização da carga, corroborando os nossos achados, no qual apenas três sessões de teste foram suficientes para encontrar a medida da carga máxima de forma acurada para esses exercícios. De maneira semelhante, Soares-Caldeira et al. ${ }^{13} \mathrm{em}$ estudo realizado com $27 \mathrm{mu}$ lheres destreinadas, mas com experiência prévia com treinamento de força de pelo menos seis meses verificou que eram necessários de 2 a 3 sessões de familiarização no exercício de rosca direta, confirmando mais uma vez os nossos resultados.

No presente estudo, foram necessárias três sessões de teste de 1-RM para estabilização da medida da carga máxima no exercício tríceps testa. Para o nosso conhecimento, nenhum estudo anterior analisou a familiarização ao teste de 1-RM nesse exercício, limitando a confrontação dos resultados. Por se tratar de um exercício uniarticular seria esperado que o número de sessões necessárias para a estabilização da carga fosse similar aos observados em outros exercícios uniarticulares, como a rosca direta, o que não ocorreu. É possível que a necessidade de equilibrar a barra e manter a articulação glenoumeral estabilizada próxima aos $90^{\circ}$ de flexão durante a realização do exercício tenha trazido maior complexidade para a realização da tarefa, exigindo maior tempo para familiarização ao exercício. Além disso, a capacidade de geração de torque da porção longa do tríceps pode ter sido influenciada pela variação angular e necessidade de estabilização da articulação glenoumeral, visto que trata-se de um músculo biarticular.

Em dois dos cinco exercícios (remada curvada e elevação frontal), não foi observada estabilização das cargas durante o período das quatro sessões de teste de 1-RM. Os fatores que são responsáveis pela falta de estabilização do exercício remada curvada parecem estar relacionados com a complexidade técnica do movimento, visto que este é um exercício multiarticular, realizado com o tronco inclinado e envolvendo grande sobrecarga, dificultando assim a estabilização da carga ${ }^{3,10}$. Segundo estudos prévios ${ }^{18,19}$ a execução de exercícios com pesos livres em situações em que se diminui o número de pontos de apoio, caso semelhante à remada curvada, proporciona maior deslocamento do centro de gravidade, maior instabilidade, aumenta o número de graus de liberdade de movimento e influencia na atividade neuromuscular, contribuindo diretamente na capacidade de geração de força muscular e consequentemente nos valores de 1-RM. Por se tratar de uma tarefa não muito comum, visto que esse exercício é geralmente executado em máquinas, é possível especular que o processo de aprendizagem e controle motor do movimento tenha colaborado para maior variação dos valores de 1-RM ao longo dos diferentes dias de teste, sendo necessárias mais sessões para sua familiarização. Em contrapartida, a condição instável da tarefa parece não explicar os resultados encontrados no exercício de elevação frontal, pois além de envolver baixa sobrecarga, durante sua execução a postura é estabilizada em uma parede. Uma possível explicação para a não estabilização da carga, é que o exercício era realizado com sobrecarga muito baixa e a inclusão de apenas dois quilos possa ter representado um incremento grande mediante a magnitude total da carga empregada. Essa hipótese é reforçada pela análise da média das diferenças entre as sessões de testes 
que em nenhum momento foi superior a um quilograma, no entanto, um quilograma nesse exercício representava quase $5 \%$ da carga total levantada (dados não apresentados).

Por outro lado, embora o exercício elevação frontal seja classificado como uniarticular, sabe-se que durante a elevação do braço é necessário o movimento coordenado das articulações glenoumeral e escapulotorácica. Alterações no posicionamento e movimentação da escápula podem alterar o recrutamento muscular e proporcionar déficits da função motora ${ }^{20}$. Nesse sentido, frente à inserção de sobrecarga relativamente alta durante o exercício, pode ter ocorrido como estratégia compensatória, alterações na movimentação das escápulas e a modificação do padrão de ativação dos músculos escapulotorácicos, influenciando, portanto no processo de estabilização dos valores de 1-RM.

Embora todos os homens incluídos neste estudo tenham relatado não realizar exercícios de força há pelo menos quatro meses, o tempo em que cada indivíduo estava sem realizar treinamento de força, bem como o tempo de experiência com o treinamento de força não foi controlado. Dessa forma, é possível que no estudo tenham sido incluídos sujeitos que estavam sem praticar treinamento de força há um longo período. Outra limitação do presente estudo é que o incremento de carga não foi padronizado em termos percentuais entre os exercícios, o que pode ter influenciado no resultado.

Sendo assim, concluísse que os resultados deste estudo revelaram a necessidade de realização de testes múltiplos de 1-RM para a avaliação acurada da carga máxima com pesos livres em homens jovens. Assim, sugere-se a realização de três sessões de teste para os exercícios supino horizontal, rosca direta de bíceps e tríceps testa e mais de quatro sessões para os exercícios de remada curvada e elevação frontal.

\section{Contribuição dos autores}

- Paulo Fernando Marinho de Lima: Coleta dos dados, análise dos dados, redação do artigo.

- Rodrigo Cappato de Araújo: Análise dos dados, redação do artigo.

- Breno Quintella Farah: Coleta dos dados, análise dos dados, redação do artigo.

- Bruno Remígio Cavalcante: Análise dos dados, redação do artigo.

- Marcos André Moura dos Santos: Análise dos dados, redação do artigo.

- Raphael Mendes Ritti Dias: Orientação do trabalho, coleta dos dados, análise dos dados, redação do artigo.

\section{REFERÊNCIAS}

1. American College of Sports Medicine. ACSM position stand. Progression models in resistance training for healthy adults. Med Sci Sports Exerc. 2009 Mar;41(3):687-708.

2. Rydwik E, Karlsson C, Frandin K, Akner G. Muscle strength testing with one repetition maximum in the arm/shoulder for people aged $75+-$ test-retest reliability. Clin Rehabil. 2007 Mar;21(3):258-65.

3. Cronin JB, Henderson ME. Maximal strength and power assessment in novice weight trainers. J Strength Cond Res. 2004 Feb;18(1):48-52.

4. Ritti-Dias RM, Cyrino ES, Salvador EP, Soares-Caldeira LF, Nakamura FY, Papst RR, et al. Influência do processo de familiarização para avaliação da força muscular em testes de 1-RM. Rev Bras Med Esporte. 2005;11(1).

5. Kraemer WJ, Fry AC. Strength testing: Development and evaluation of methodology. In Physiological assessment of human fitness. Chapaingn IHK, editor. In Physiological assessment of human fitness.1995 1995 . 
6. Fleiss JL. Design and Analysis of Clinical Experiments. Wiley Classics Library: WileyInterscience; 1999. p. 448.

7. Benton MJ, Swan PD, Peterson MD. Evaluation of multiple one repetition maximum strength trials in untrained women. J Strength Cond Res. 2009 Aug;23(5):1503-7.

8. Clarke DH. Adaptations in strength and muscular endurance resulting from exercise. Exerc Sport Sci Rev. 1973;1:73-102.

9. Dias RMR, Avelar A, Salvador EP, Cyrino ES. Familiarização ao teste de 1-RM em mulheres com experiência prévia em treinamento com pesos. Rev Educ Fis/UEM. 2009;20(3):423-9.

10. Dias RMR, Cyrino ES, Salvador EP, Caldeira LFS, Nakamura FY, Papst RR, et al. Influência do processo de familiarização para avaliação da força muscular em testes de 1-RM. Rev Bras Med Esporte. 2005;11:34-8.

11. Gurjão ALD, Cyrino ES, Soares-Caldeira LF, Nakamura FY, Oliveira ARd, Salvador EP, et al. Variação da força muscular em testes repetitivos de 1-RM em crianças pré-púberes. Rev Bras Med Esporte. 2005;11(6).

12. Ritti-Dias RM, Avelar A, Salvador EP, Cyrino ES. Influence of previous experience on resistance training on reliability of one-repetition maximum test. J Strength Cond Res. 2011 May;25(5):1418-22.

13. Soares-Caldeira LF, Ritti-Dias RM, Okuno NM, Cyrino ES, Gurjao AL, Ploutz-Snyder LL. Familiarization indexes in sessions of 1-RM tests in adult women. J Strength Cond Res. 2009 Oct;23(7):2039-45.

14. Gurjão ALD, Cyrino ES, Caldeira LFS, Nakamura FY, Oliveira ARd, Salvador EP, et al. Variação da força muscular em testes repetitivos de 1-RM em crianças pré-púberes. Rev Bras Med Esporte. 2005;11:319-24.

15. Ploutz-Snyder LL, Giamis EL. Orientation and familiarization to $1 R M$ strength testing in old and young women. J Strength Cond Res. 2001 Nov;15(4):519-23.

16. Levinger I, Goodman C, Hare DL, Jerums G, Toia D, Selig S. The reliability of the 1RM strength test for untrained middle-aged individuals. J Sci Med Sport. 2009 Mar;12(2):310-6.

17. Phillips WT, Batterham AM, Valenzuela JE, Burkett LN. Reliability of maximal strength testing in older adults. Arch Phys Med Rehabil. 2004 Feb;85(2):329-34.

18. Saeterbakken AH, Fimland MS. Effects of body position and loading modality on muscle activity and strength in shoulder presses. J Strength Cond Res. 2013 Jul;27(7):1824-31.

19. Saeterbakken AH, van den Tillaar R, Fimland MS. A comparison of muscle activity and 1-RM strength of three chest-press exercises with different stability requirements.J Sports Sci. 2011 Mar;29(5):533-8.

20. Oliveira VMA, Batista LdSP, Pirauá ALT, Pitangui ACR, Araújo RCd. Electromyographic activity and scapular dyskenesia in athletes with and without shoulder impingement syndrome. Rev Bras Cineantropom Desempenho Humano. 2013;15:193-203.

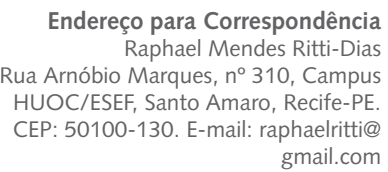

